\title{
N-TYPE THERMOELECTRIC TEXTILE FABRICS BASED CIWC-2 ON VAPOR GROWN CARBON NANOFIBERS
}

\author{
S. Machado ${ }^{1}$, E. M. F. Vieira ${ }^{2}$, A. M. Rocha ${ }^{1}$, A. J. Paleo ${ }^{1 *}$ \\ ${ }^{12}$ C2T-Centre for Textile Science and Technology, University of Minho, Campus de Azurém, \\ Guimarães, Portugal. \\ ${ }^{2}$ CMEMS-UMinho-Center for MicroElectromechanical Systems, University of Minho, \\ Campus de Azurém, Guimarães, Portugal. \\ *ajpaleovieito@2c2t.uminho.pt
}

\section{Introduction}

Conductive fillers like carbon nanotubes (CNT) are extensively investigated to fabricate thermoelectric (TE) materials. Nevertheless, most as-produced CNT have positive Seebeck Coefficients due to oxygen doping (Figure 1). It is for this reason that similar carbon nanostructures like vapor grown carbon nanofibers (CNF) grown by chemical vapor deposition (CVD) can fill the current lack of simple pathways towards the direct production of n-type TE materials.

\section{Methods}

Three aqueous dispersions of Pyrograf ${ }^{\oplus} I I I$ PR 25 PS XT with a pyrolitically outer stripped layer and diameters around $115 \mathrm{~nm}$, grown by CVD at $1100{ }^{\circ} \mathrm{C}$, were used for coating $1 / 1$ plain cotton woven fabrics of $14.9 \times 20.2$ Tex by dipping-drying process (Figure 2). Their electrical conductivity $(\sigma)$ was tested by conventional four probe van der Pauw method, and their Seebeck coefficient (S) by using a setup system for a parallel (in-plane) measurement [2].

\section{Results}

The conductivity of the dip-coated cotton fabrics depends strongly on the concentration of CNF dispersions used. Samples CWF@1.6CNF and CWF@3.2CNF showed $\sigma$ values of 8 and $17 \mathrm{~S} \mathrm{~m}^{-1}$, respectively, and similar negative Seebeck coefficients of around $-10 \mu \mathrm{VK}^{-1}$, whereas samples CWF@6.4CNF dip-coated with higher content of CNF dispersion $(6.4 \mathrm{mg} / \mathrm{ml})$ achieved the highest $\sigma$ and $\mathrm{S}$ of $46 \mathrm{~S}$ $\mathrm{m}^{-1}$ and $-12 \mu \mathrm{VK}^{-1}$, respectively.

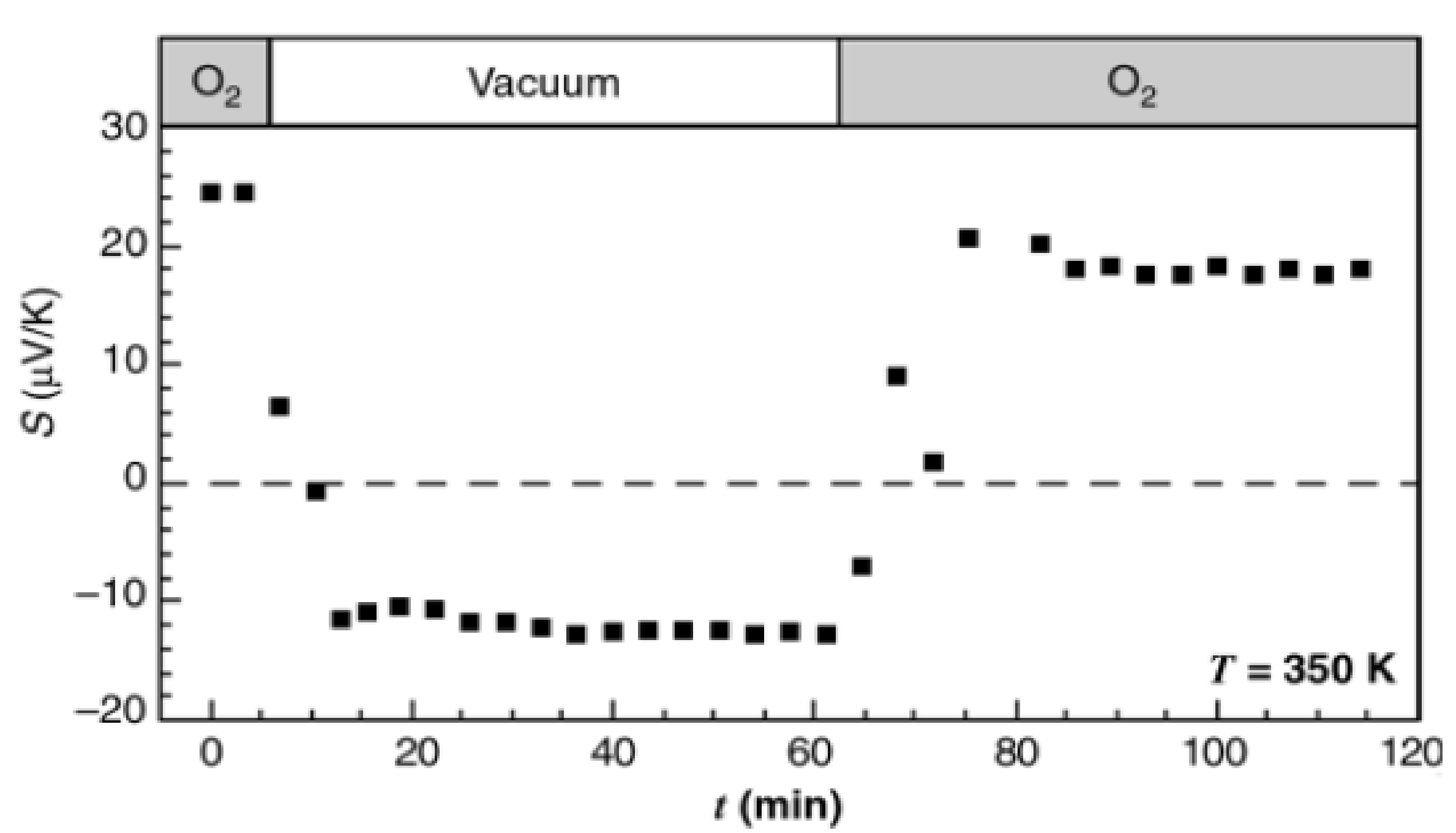

Figure 1. Sensitivity to environmental conditions of thermoelectric power $S$ for SWCNTs at $T=350 \mathrm{~K}$ [1].

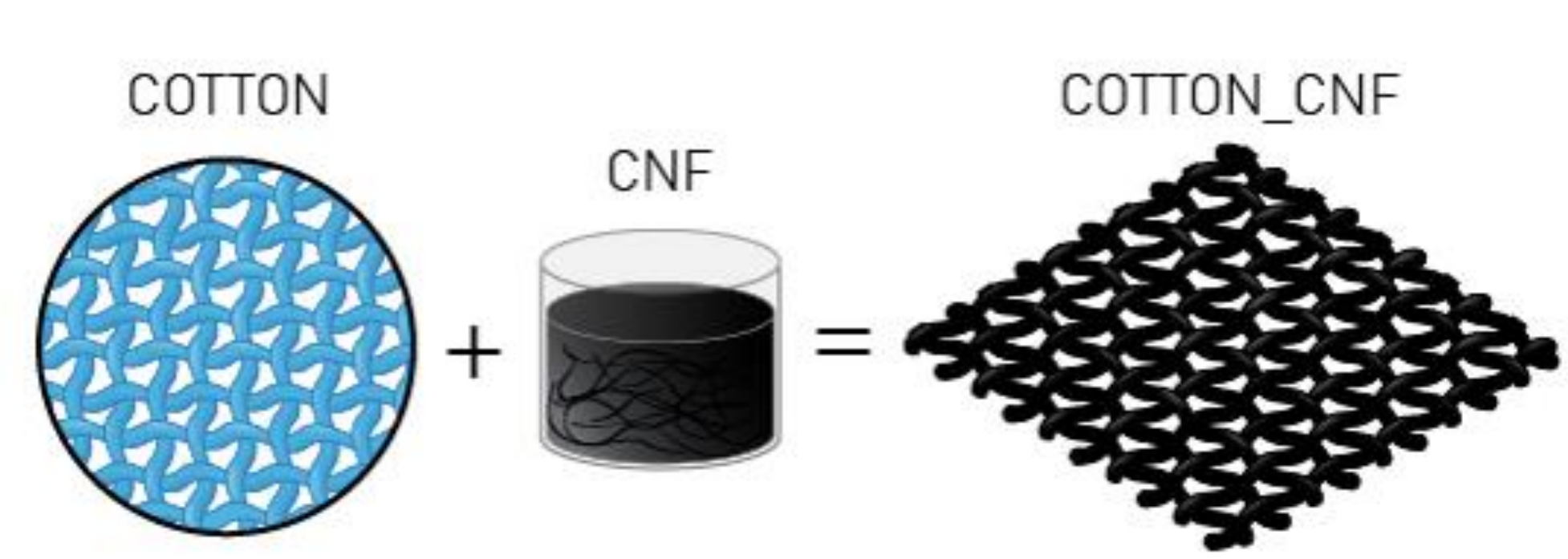

\begin{tabular}{|c|c|c|c|}
\hline Sample & $\begin{array}{l}\text { CNF aqueous } \\
\text { dispersion } \\
(\mathrm{mg} / \mathrm{ml})\end{array}$ & CNF & $\begin{array}{l}\text { Cotton fabric linear density } \\
\text { (Tex) }\end{array}$ \\
\hline CWF@1.6CNF & 1.6 & \multirow{3}{*}{ PR 25 PS XT } & \multirow{3}{*}{$14.9 \times 20.2$} \\
\hline CWF@3.2CNF & 3.2 & & \\
\hline CWF@6.4CNF & 6.4 & & \\
\hline
\end{tabular}

Figure 2. Dip-coating processing. (Table) Dip-coated fabric sample type.

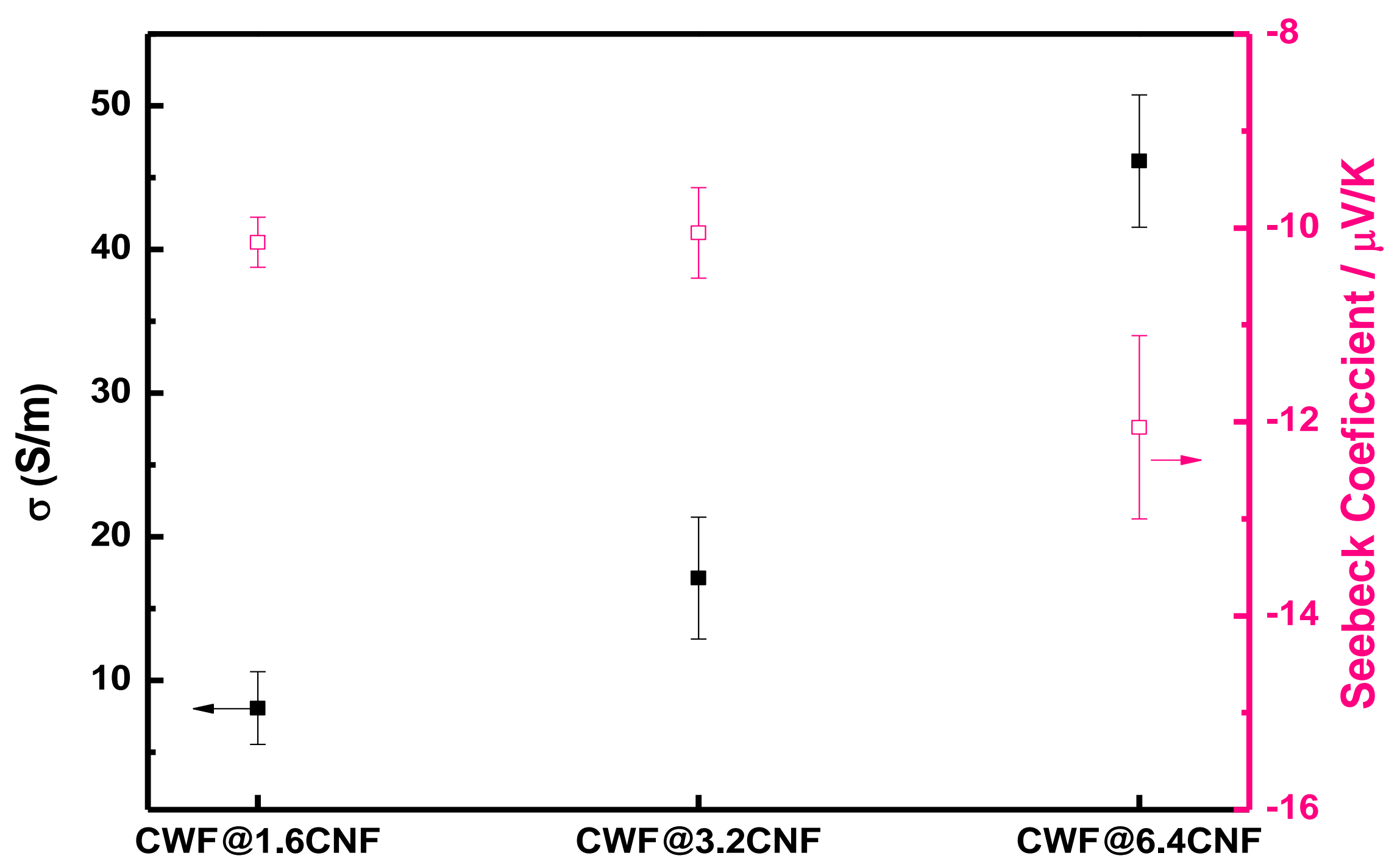

Figure 3. Electrical conductivity (solid symbols), and negative Seebeck (open symbols) of dip-coated cotton fabrics at room temperature.

\section{Conclusion}

The negative Seebeck of cotton fabrics based on vapor grown carbon nanofibers means that the majority of their charge carriers are electrons, in contrast with most asproduced CNT that show positive $S$ due to their immediate oxygen doping after synthesis. We attribute this negative $S$ to the double wall structure surrounding the hollow tube of the CNF. The n-type contribution caused by the highly graphitic character of the inner shells must counteract the -

lower p-type contribution caused by the disordered and thinner outer shells [3]. These results show that commercial and as-received CNF can be used for fabricating directly N-type TE textile fabrics, without requiring deoxygenation pre-treatments and/or further specific additives during their processing.

[1] A. Zettl, Science, $287(2000)$ 1801-1804.

[2] O. Okhay, G. Gonçalves, A. Tkach, C. Dias, J. Ventura, M.F. Ribeiro Da Silva, L.M. Valente Gonçalves, E. Titus, Journal of Applied Physics, 120 (2016).

[3] A.J. Paleo, E.M.F. Vieira, K. Wan, O. Bondarchuk, M.F. Cerqueira, L.M. Goncalves, E. Bilotti, P. Alpuim, A.M. Rocha, Carbon, 150 (2019) 408-416.

\section{Acknowledgments}

This work was financed by FEDER funds through COMPETE and by national funds through FCT Foundation for Science and Technology within the project POCI-01-0145-FEDER-007136. E. M. F. Vieira is grateful for financial support through FCT with CMEMS-UMinho Strategic Project UIDB/04436/2020.

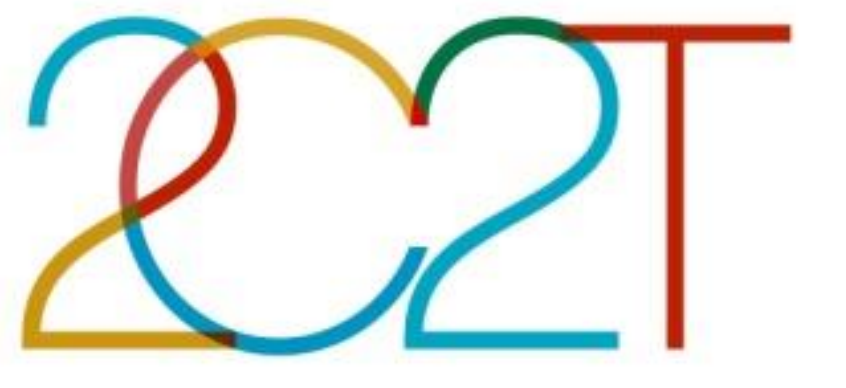

